\title{
Impact of Decision Rules and Non-Cooperative Behaviors on Minimum Consensus Cost in Group Decision Making
}

\author{
Weijun Xu ${ }^{\mathrm{a}}$, Xin Chen ${ }^{\mathrm{a}}$, Yucheng Dong ${ }^{\mathrm{b}}$, Francisco Chiclana ${ }^{\mathrm{c}, \mathrm{d}}$ \\ ${ }^{\text {a }}$ School of Business Administration, South China University of Technology, Guangzhou \\ 510641, China \\ ${ }^{\mathrm{b}}$ Business School, Sichuan University, Chengdu 610065, China \\ c Institute of Artificial Intelligence, School of Computer Science and Informatics, \\ De Montfort University, Leicester, United Kingdom \\ d Andalusian Research Institute in Data Science and Computational Intelligence, University of \\ Granada, Granada, Spain
}

Email addresses: xuwj@scut.edu.cn (W.Xu), chenxin_bjfu@163.com (X. Chen),ycdong@scu.edu.cn (Y. Dong), chiclana@dmu.ac.uk (F. Chiclana).

\section{Acknowledgement}

Weijun $\mathrm{Xu}$ would like to acknowledge the financial support of grants (Nos. 71771091, 71471065) from NSF of China, and Yucheng Dong would like to acknowledge the financial support of grant (No. 71871149) from NSF of China, and grant (No. sksyl201705) from Sichuan University. 


\title{
Impact of Decision Rules and Non-Cooperative Behaviors on Minimum Consensus Cost in Group Decision Making
}

\begin{abstract}
In group decision making (GDM), it is sensible to achive minimum consensus cost (MCC) because the consensus reaching process (CRP) resources are often limited. In this endeavour, though, there are still two issues that require paying attention to: (1) the impact of decision rules, including decision weights and aggregation functions, on MCC; and (2) the impact of non-cooperative behaviors on MCC. Hence, this paper analytically reveals the decision rules to minimize MCC or maximize MCC. Furthermore, detailed simulation experiments show the joint impact of non-cooperative behavior and decisions rules on MCC, as well as revealing the effect of the consensus within the established MCC target.
\end{abstract}

Keywords: Group decision making; Consensus; Cost; Decision rules; Non-cooperative behaviors; Simulation experiment.

\section{Introduction}

Group decision-making (GDM) can be viewed as a process where a group of decision makers express their opinions and aim at achieving a collective solution. Consensus reaching process (CRP) is a key issue in GDM by which decision makers are assisted to achieve consensus regarding certain collective solution. Traditionally, "hard" consensus in GDM considers only a lack of consensus state or a state of full or unanimous, which is inconvenient and unnecessary in real life (Kacprzyk et al., 1997, 2010; Kacprzyk \& Zadrożny, 2010, 2016). Thus, different consensus reaching processes (CRPs) based on a "soft" consensus measure in GDM have been proposed, with some excellent reviews on CRP available in (Herrera-Viedma et al., 2014; Palomares et al., 2014a). In particular, the minimum consensus cost (MCC) and non-cooperative behaviors are becoming hot topics in CRPs.

Generally, preference changing are associated a cost and the resources for consensus building are limited (Ben-Arieh \& Easton, 2007; Dong et al., 2010, 2015; Dong \& Xu, 2016; Gong et al., 2017, 2019; Tan et al., 2018 ). Based on these premises, some CRPs with MCC have been developed (Ben-Arieh \& Easton, 2007; Ben-Arieh et al., 2008; Cheng et al., 2018; Gong et al., 2015a, 2015b; Zhang et al., 2017, 2019b). Notably, Zhang et al. (2019c) investigated the consensus efficiency of existing CRPs with MCC and provided detailed simulation experiments based on the following comparison criteria: the number of adjusted decision makers; the number of adjusted alternatives; the number of adjusted preference values; the distance between the original and the adjusted preference information; and the number of negotiation rounds required to reach consensus.

In GDM problems, decision makers may behave uncooperatively by expressing dishonest opinions or refusing to change their opinions to favor their own profit. Two mainstream 
research approaches have been developed in the literature to effectively address non-cooperative behaviors and ensure the quality of the GDM results: (1) managing non-cooperative behaviors in the aggregation process or selection process in the GDM, which focuses on the influence of the non-cooperative behaviors on the aggregation outcome (Dong et al., 2017; Pelta \& Yager, 2010; Yager, 2001, 2002); (2) managing non-cooperative behaviors in the consensus process of the GDM, which mainly analyzes whether a consensus solution can be achieved under the presence of non-cooperative behaviors in the CRP (Dong et al., 2016, 2018b; Palomares et al., 2014b; Quesada et al., 2014).

Although numerous studies have been presented to analyze the MCC and non-cooperative behaviors in CRPs, there still exist two issues that need to be dealt with: the impact of the decision rules and of non-cooperative behaviors on MCC.

(i) Impact of decision rules on MCC. In existing CRP studies, decision rules include decision weights and aggregation functions. It is natural that setting decision rules will lead to different consensus outcomes and will have an effect on the consensus cost. For example, in the selection of outstanding research projects, choosing different aggregation functions and decision weights can lead to different consensus outcome and cost. However, the existing research about MCC is to develop some consensus models under the given decision rules and decision weights, and it is still not clear how decision rules influence the minimum cost in reaching consensus in GDM.

(ii) Impact of non-cooperative behaviors on MCC. Although non-cooperative behaviors have been extensively studied, the existing research about non-cooperative behaviors focuses on the mangement of non-cooperative behaviors in CRPs, and it is still unclear how non-cooperative behaviors influence on the MCC in CRPs. Therefore, it is necessary to reveal the internal mechanism of the non-cooperative behaviors impact on MCC.

This paper focuses on these two issues and it presents the following research contributions on the impact of decision rules and non-cooperative behaviors on MCC in GDM:

(i) From a theoretical point of view, it is proved that the decision rule that minimizes MCC can be modeled with the ordered weighted average (OWA) with decision weigh $w=$ $(0.5,0, \ldots, 0,0.5)^{T}$, while the decision rule that maximize the MCC is modeled with the OWA with decision weight $w=(1,0, \ldots, 0)^{T}$ or $w=(0, \ldots, 0,1)^{T}$.

(ii) From an analytical point of view, Simulation experiments I, II and III are designed to show that the non-cooperative behaviors strongly increase the MCC. The MCC increases with an increase on the number of decision makers. This positive relationship is more obvious when decision makers are less tolerant to inconsistent views or the non-cooperative behaviors in the group are high. We also show non-cooperative behaviors is a more determiner factor in influencing the MCC than decision rules. 
Furthermore, the effect of the consensus within the established target on MCC is also studied in the simulation experiments.

The rest of this paper is organized as follows: Section 2 describes the general CRP framework and the minimum-cost consensus model. Section 3 is devoted to the theoretical study to reveal of decision rules impact on MCC. Section 4 provides an analytical study with Simulation experiment I, II and III to further reveals the impact of non-cooperative behaviors and decision rules on MCC. Lastly, Section 5 concludes the paper.

\section{Background}

This section briefly describes the general CRP framework and the minimum-cost consensus model, which will provide the basis of this study.

\subsection{The general CRP framework}

The general CRP framework is depicted in Fig. 1 (Herrera-Viedma et al., 2002; Zhang et al., 2011). Once the individual experts provide their opinions, an aggregation function is carried out to derive the collective opinion. Then, the consensus level among the group of decision makers is measured by looking at the difference between the individual opinions and the collective opinion. If the consensus level is lower than an apriority acceptable consensus threshold value, then a feedback process is carried out to provide support to the decision makers to adjust their individual opinions with the aim of increasing the group consensus. This procedure is repeated for a maximum number of consensus rounds or until the group consensus level reaches the threshold level, whichever comes first.

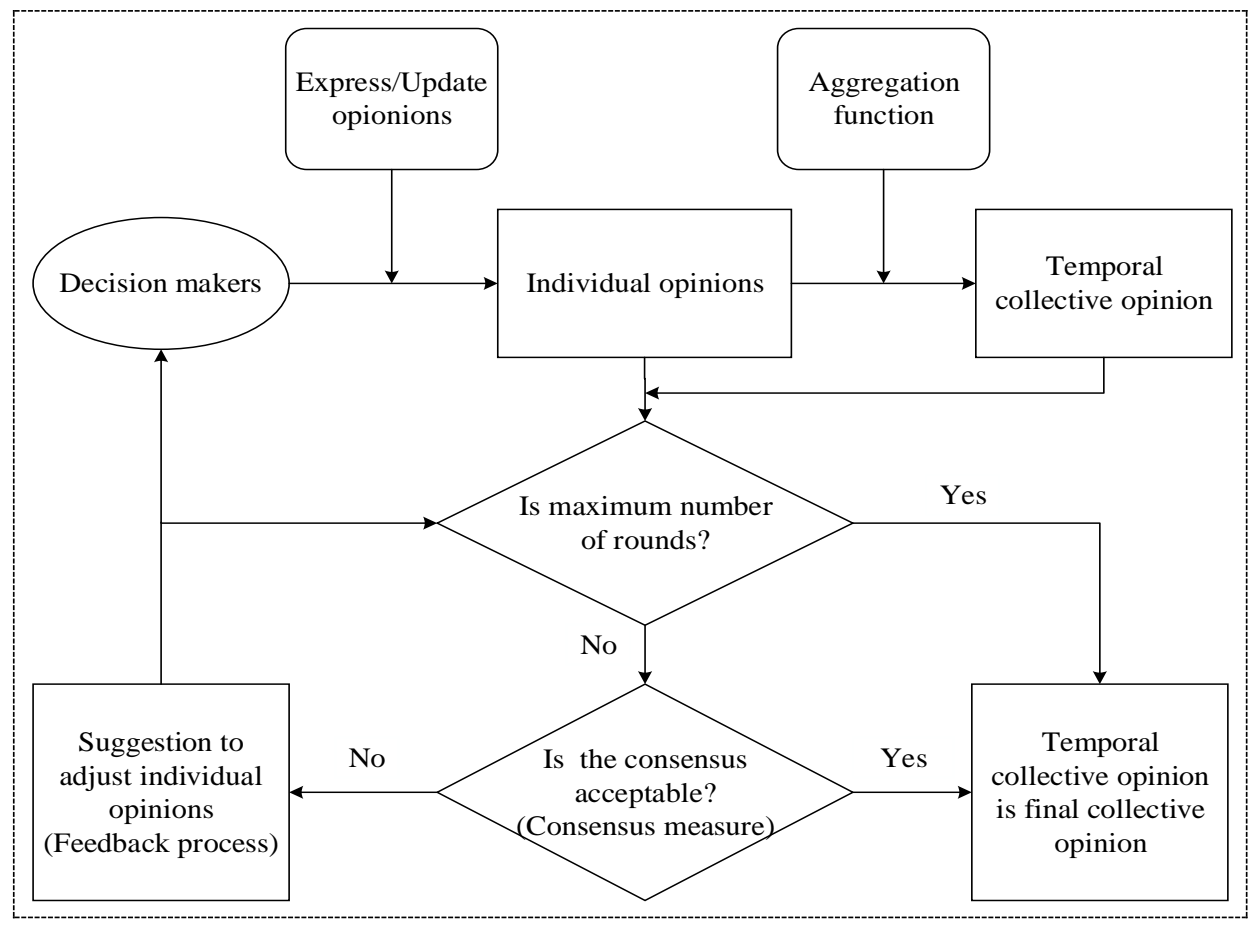

Fig. 1 Framework of the general CRP. 


\subsection{Minimum-Cost Consensus Model}

In a GDM problem, let $D=\left\{d_{1}, d_{2}, \ldots, d_{n}\right\}$ be the set of decision makers, $W=$ $\left(w_{1}, w_{2}, \ldots, w_{n}\right)^{T}$ the associated decision makers weights vector, where $w_{i} \geq 0(i=$ $1,2, \ldots, n)$ and $\sum_{i=1}^{n} w_{i}=1 ; \quad A=\left\{a_{1}, a_{2}, \ldots, a_{n}\right\}$ be the set of individual opinions, where $a_{i} \in[0,1]$ represents the decision maker $d_{i}$ 's opinion on an alternative.

The minimum-cost consensus model includes three parts:

(1) Consensus measurement. Several methods can be used to measure the consensus level in a GDM problem (Fedrizzi, 1988; Kacprzyk et al., 1922). The most common is based on the use of a distance based measure, which is the one employed in the minimum-cost consensus model presented in (Chiclana et al., 2013). Let the collective opinion be represented by $a^{c}$. The consensus level of the decision maker $d_{i}$ can be measured using the value $\left|a_{i}-a^{c}\right|$ $(i=1,2, \ldots, n)$. Let $\varepsilon$ be a threshold value (Herrera-Viedma et al., 2002). If

$$
\left|a_{i}-a^{c}\right| \leq \varepsilon, \text { for all } i=1,2, \ldots, n,
$$

then all decision makers are considered to have reached an acceptable consensus. Otherwise, the decision makers need to adjust their opinions to increase the consensus level.

(2) Consensus cost. Let $\overline{a_{i}} \in \bar{A}$ denote the adjusted individual opinion of decision maker $d_{i}$, and let $\overline{a^{c}}$ be the adjusted collective opinion. Ben-Arieh and Easton (2007) assumed that moving $d_{i}$ 's opinion 1 unit has associated a cost $c_{i} \in[0,+\infty)$, and defined the linear consensus cost of moving $d_{i}$ 's opinion from $a_{i}$ to $\overline{a_{i}}$ as $c_{i}\left|a_{i}-\overline{a_{i}}\right|$. Thus, it is natural to minimize the consensus cost, i.e.,

$$
\min _{\overline{a_{i}}} \sum_{i=1}^{n} c_{i}\left|a_{i}-\overline{a_{i}}\right|
$$

(3) Aggregation function. In GDM problems, aggregation functions are used to fuse individuals' opinions to form a collective opinion (Akram et al., 2018, 2019a, 2019b, 2019c; Dong et al., 2010; Ogryczak \& Śliwiński, 2003; Zhang et al., 2019a). The weighted average (WA) and ordered weighted average (OWA) operators are the most important aggregation functions in GDM problems (Dong et al., 2010; Ogryczak \& Śliwiński, 2003; Zhang et al., 2011), which are defined by Eqs. (3) and (4), respectively.

$$
\begin{gathered}
F_{W A}\left(a_{1}, a_{2}, \ldots, a_{n}\right)=\sum_{i=1}^{n} w_{i} a_{i}, \\
F_{O W A}\left(a_{1}, a_{2}, \ldots, a_{n}\right)=\sum_{i=1}^{n} w_{i} a_{(i)},
\end{gathered}
$$

where $a_{(i)}$ is the $i$ th largest element of $A=\left\{a_{1}, a_{2}, \ldots, a_{n}\right\}$, and $W=\left(w_{1}, w_{2}, \ldots, w_{n}\right)^{T}$ is the associated weight vector.

In minimum-cost consensus model, the WA and OWA operators are employed to aggregate individuals' opinion to obtain the collective decision option $a^{c}$,i.e.,

$$
a^{c}=F_{W}\left(a_{1}, a_{2}, \ldots, a_{n}\right) .
$$


Formally, Zhang et al. (2011) proposed the following minimum-cost consensus model with aggregation functions:

$$
\left\{\begin{array}{c}
\min _{\overline{a_{i}}} \sum_{i=1}^{n} c_{i}\left|a_{i}-\overline{a_{i}}\right| \\
\overline{a^{c}}=F_{W}\left(\overline{a_{1}}, \overline{a_{2}}, \ldots, \overline{a_{n}}\right) \\
\left|\overline{a_{i}}-\overline{a^{c}}\right| \leq \varepsilon, i=1,2, \ldots, n
\end{array} .\right.
$$

In this study, we denote this model as $P_{1}$. Solving $P_{1}$ yields the optimal adjusted opinions. Similarly, the respective minimum-cost consensus models with aggregation functions OWA and WA, denoted models $P_{2}$ and $P_{3}$, respectively, are:

$$
\begin{gathered}
\left\{\begin{array}{c}
\min _{\overline{a_{i}}} \sum_{i=1}^{n} c_{i}\left|a_{i}-\overline{a_{i}}\right| \\
\overline{a^{c}}=F_{O W A}\left(\overline{a_{1}}, \overline{a_{2}}, \ldots, \overline{a_{n}}\right), \\
\left|\overline{a_{i}}-\overline{a^{c}}\right| \leq \varepsilon, i=1,2, \ldots, n
\end{array}\right. \\
\left\{\begin{array}{c}
\min _{\overline{a_{i}}} \sum_{i=1}^{n} c_{i}\left|a_{i}-\overline{a_{i}}\right| \\
\overline{a^{c}}=F_{W A}\left(\overline{a_{1}}, \overline{a_{2}}, \ldots, \overline{a_{n}}\right) . \\
\left|\overline{a_{i}}-\overline{a^{c}}\right| \leq \varepsilon, i=1,2, \ldots, n
\end{array}\right.
\end{gathered}
$$

\section{Impact of decision rules on minimum consensus cost}

In a CRP, the aggregation function and its associated decision weight play the role of the decision rules. This section reveals the impact of decision rules on MCC.

\subsection{The decision rule to minimize MCC}

The solution of the model $P_{4}$ below will be the optimal adjusted collective opinion that minimizes the MCC.

$$
\left\{\begin{array}{l}
\frac{\min }{a^{c}} \min _{\overline{a_{i}}} \sum_{i=1}^{n} c_{i}\left|a_{i}-\overline{a_{i}}\right| \\
\left|\overline{a_{i}}-\overline{a^{c}}\right| \leq \varepsilon, i=1,2, \ldots, n
\end{array} .\right.
$$

Let model $P_{5}$ be defined by Eq. (10):

$$
\begin{gathered}
\min _{\overline{a_{i}}} \sum_{i=1}^{n} c_{i}\left|a_{i}-\overline{a_{i}}\right| \\
\overline{a^{c}}=\left(\min _{i} \overline{a_{i}}+\max _{i} \overline{a_{i}}\right) / 2 . \\
\left(\left|\overline{a_{i}}-\overline{a^{c}}\right| \leq \varepsilon, i=1,2, \ldots, n\right.
\end{gathered}
$$

The following results show that the optimal solution of model $P_{5}$ is also the optimal solution of model $P_{4}$.

Theorem 1: Let $\overline{a_{i}^{*}}(i=1,2, \ldots, n)$ and $\overline{a^{c *}}$ denote the optimal adjusted individual opinions and adjusted collective opinion obtained by solving model $P_{5}$. Then, 
$\left\{\overline{a_{1}^{*}}, \overline{a_{2}^{*}}, \ldots, \overline{a_{n}^{*}}, \overline{a^{c *}}\right\}$ is the optimal solution of model $P_{4}$.

\section{Proof:}

Let $\left\{\overline{\overline{a_{1}}}, \overline{\overline{a_{2}}}, \ldots, \overline{\overline{a_{n}}}, \overline{\overline{a^{c}}}\right\}$ be the optimal solution of model $P_{4}$, and $\Omega_{4}$ its feasible set. Similarly, let $\left\{\overline{a_{1}^{*}}, \overline{a_{2}^{*}}, \ldots, \overline{a_{n}^{*}}, \overline{a^{c *}}\right\}$ be the optimal solution of model $P_{5}$, and $\Omega_{5}$ its feasible set.

Based on the condition $\left|\overline{a_{i}}-\overline{a^{c}}\right| \leq \varepsilon(i=1,2, \ldots, n)$ of model $P_{4}$, then we would get

$$
\min _{\overline{a_{i}}} \sum_{i=1}^{n} c_{i}\left|a_{i}-\overline{a_{i}}\right|=\frac{\min }{a^{c}}\left[\sum_{i: a_{i}<\overline{a^{c}}-\varepsilon} c_{i}\left(\overline{a^{c}}-\varepsilon-a_{i}\right)+\sum_{i: a_{i}>\overline{a^{c}}+\varepsilon} c_{i}\left(a_{i}-\varepsilon-\overline{a^{c}}\right)\right]
$$

Also because

$$
\begin{aligned}
& \frac{\min }{a^{c}} \min _{\overline{a_{i}}} \sum_{i=1}^{n} c_{i}\left|a_{i}-\overline{a_{i}}\right| \\
= & \min _{\frac{a^{c}}{a^{c}}} \frac{\min }{a^{c}}\left[\sum_{i: a_{i}<\overline{a^{c}}-\varepsilon} c_{i}\left(\overline{a^{c}}-\varepsilon-a_{i}\right)+\sum_{i: a_{i}>\overline{a^{c}}+\varepsilon} c_{i}\left(a_{i}-\varepsilon-\overline{a^{c}}\right)\right] \\
= & \min _{\overline{a^{c}}}\left[\sum_{i: a_{i}<\overline{a^{c}}-\varepsilon} c_{i}\left(\overline{a^{c}}-\varepsilon-a_{i}\right)+\sum_{i: a_{i}>\overline{a^{c}}+\varepsilon} c_{i}\left(a_{i}-\varepsilon-\overline{a^{c}}\right)\right]
\end{aligned}
$$

Then it will be

$$
\frac{\min }{a^{c}} \min _{a_{i}} \sum_{i=1}^{n} c_{i}\left|a_{i}-\overline{a_{i}}\right|=\min _{\overline{a_{i}}} \sum_{i=1}^{n} c_{i}\left|a_{i}-\overline{a_{i}}\right| .
$$

Therefore, model $P_{4}$ is equivalent to Eq. (14)

$$
\left\{\begin{array}{l}
\min _{\overline{a_{i}}} \sum_{i=1}^{n} c_{i}\left|a_{i}-\overline{a_{i}}\right| \\
\left|\overline{a_{i}}-\overline{a^{c}}\right| \leq \varepsilon, i=1,2, \ldots, n
\end{array} .\right.
$$

Thus, $\left\{\overline{\overline{a_{1}}}, \overline{\overline{a_{2}}}, \ldots, \overline{\overline{a_{n}}}, \overline{\overline{a^{c}}}\right\}$ and $\Omega_{4}$ also represent the optimal solution and the feasible set of Eq. (14). Therefore, $\Omega_{5} \subseteq \Omega_{4}$, and we have that:

$$
\begin{aligned}
\sum_{i=1}^{n} c_{i}\left|a_{i}-\overline{\bar{a}}_{i}\right| & =\min _{\left\{\overline{a_{1}}, \overline{a_{2}}, \ldots, \overline{a_{n}}, \overline{a^{c}}\right\} \in \Omega_{4}} \sum_{i=1}^{n} c_{i}\left|a_{i}-\overline{a_{i}}\right| \\
& \leq \min _{\left\{\overline{a_{1}}, \overline{a_{2}}, \ldots, \overline{a_{n}}, \overline{a^{c}}\right\} \in \Omega_{5}} \sum_{i=1}^{n} c_{i}\left|a_{i}-\overline{a_{i}}\right| \\
& =\sum_{i=1}^{n} c_{i}\left|a_{i}-\overline{a_{i}^{*}}\right| .
\end{aligned}
$$

Moreover, the relationship between $\overline{\overline{a^{c}}}$ and $\overline{\bar{a}}_{i}$ is:

$$
\overline{\bar{a}}_{i}=\left\{\begin{array}{c}
\overline{\overline{a^{c}}}-\varepsilon, \quad i \in\left\{i: a_{i} \leq \overline{\overline{a^{c}}}-\varepsilon\right\} \\
\overline{\overline{a^{c}}}+\varepsilon, \quad i \in\left\{i: a_{i} \geq \overline{\overline{a^{c}}}+\varepsilon\right\}, \\
a_{i}, i \in\left\{i: \overline{\overline{a^{c}}}-\varepsilon<a_{i}<\overline{\overline{a^{c}}}+\varepsilon\right\}
\end{array},\right.
$$

which satisfies $\max _{i} \overline{\bar{a}}_{i}-\min _{i} \overline{\bar{a}}_{i} \leq 2 \varepsilon$.

Furthermore, it is $\left|\overline{\bar{a}}_{i}-\left[\left(\max _{i} \overline{\bar{a}}_{i}+\min _{i} \overline{\bar{a}}_{i}\right) / 2\right]\right| \leq \varepsilon$ for all $i=1,2, \ldots, n$. Therefore, $\left\{\overline{\overline{a_{1}}}, \overline{\overline{a_{2}}}, \ldots, \overline{\overline{a_{n}}},\left[\left(\max _{i} \overline{\bar{a}}_{i}+\min _{i} \overline{\bar{a}}_{i}\right) / 2\right]\right\} \in \Omega_{5}$. Consequently, 


$$
\begin{gathered}
\sum_{i=1}^{n} c_{i}\left|a_{i}-\overline{\bar{a}}_{i}\right| \geq \min _{\left\{\overline{a_{1}}, \overline{a_{2}}, \ldots, \overline{a_{n}}, \overline{a^{c}}\right\} \in \Omega_{5}} \sum_{i=1}^{n} c_{i}\left|a_{i}-\overline{a_{i}}\right| \\
=\sum_{i=1}^{n} c_{i}\left|a_{i}-\overline{a_{i}^{*}}\right| .
\end{gathered}
$$

From Eqs. (15) and (17), it is:

$$
\sum_{i=1}^{n} c_{i}\left|a_{i}-\overline{\bar{a}}_{i}\right|=\sum_{i=1}^{n} c_{i}\left|a_{i}-\overline{a_{i}^{*}}\right| .
$$

Thus, $\left\{\overline{a_{1}^{*}}, \overline{a_{2}^{*}}, \ldots, \overline{a_{n}^{*}}, \overline{a^{c *}}\right\}$ is the optimal solution to model $P_{4}$.

This completes the proof of Theorem 1.

In the following, we further derive the decision rule between the optimal adjusted collective opinion and the optimal individual opinions of model $P_{4}$. Based on Theorem 1, $\left\{\overline{a_{1}^{*}}, \overline{a_{2}^{*}}, \ldots, \overline{a_{n}^{*}}, \overline{a^{c *}}\right\}$ is the optimal solution of model $P_{4}$, which still satisfies the functional relationship $\overline{a^{c *}}=\left(\min _{i} \overline{a_{i}^{*}}+\max _{i} \overline{a_{i}^{*}}\right) / 2$ of model $P_{5}$, i.e. the decision rule that minimizes the MCC of model $P_{4}$ is:

$$
\overline{a^{c}}=F_{O W A}\left(\overline{a_{1}}, \overline{a_{2}}, \ldots, \overline{a_{n}}\right),
$$

with decision weight vector

$$
O W A \_\min =(0.5,0, \ldots, 0,0.5)^{T} .
$$

\subsection{The decision rule to maximize MCC}

Solving the next model $P_{6}$ will lead to the optimal adjusted collective opinion that maximizes the MCC.

$$
\left\{\begin{array}{l}
\frac{\max }{a^{c}} \min \sum_{i=1}^{n} c_{i}\left|a_{i}-\overline{a_{i}}\right| \\
\left|\overline{a_{i}}-\overline{a^{c}}\right| \leq \varepsilon, i=1,2, \ldots, n
\end{array} .\right.
$$

First, we provide the following result regarding the optimal solution of model $P_{6}$

Lemma 1: Let $\left\{o_{1}, o_{2}, \ldots, o_{n}, o^{c}\right\}$ denote the optimal solution of model $P_{1}$. Then, it is:

$o^{c} \in\left[\min _{i} a_{i}, \max _{i} a_{i}\right]$.

\section{Proof:}

Since $\left\{o_{1}, o_{2}, \ldots, o_{n}, o^{c}\right\}$ is the optimal solution of model $P_{1}$. Let $\left\{b_{1}, b_{2}, \ldots, b_{n}, b^{c}\right\}$, $\left\{e_{1}, e_{2}, \ldots, e_{n}, e^{c}\right\},\left\{f_{1}, f_{2}, \ldots, f_{n}, f^{c}\right\}$, and $\left\{g_{1}, g_{2}, \ldots, g_{n}, g^{c}\right\}$ denote feasible solutions of model $P_{1}$, where $b^{c} \in\left[0, \min _{i} a_{i}\right), e^{c} \in\left(\max _{i} a_{i}, 1\right], f^{c}=\min _{i} a_{i}$ and $g^{c}=\max _{i} a_{i}$ and $\varepsilon$ being a small enough number.

(i) For $b^{c} \in\left[0, \min _{i} a_{i}\right)$, then

$$
M C C_{1}=\sum_{i=1}^{n} c_{i}\left|a_{i}-b_{i}\right|=\sum_{i=1}^{n} c_{i}\left(a_{i}-b_{i}\right) .
$$


(ii) For $e^{c} \in\left(\max _{i} a_{i}, 1\right]$, then

$$
M C C_{2}=\sum_{i=1}^{n} c_{i}\left|a_{i}-e_{i}\right|=\sum_{i=1}^{n} c_{i}\left(e_{i}-a_{i}\right) .
$$

(iii) For $f^{c}=\min _{i} a_{i}$, then

$$
\operatorname{MCC}_{3}=\sum_{i=1}^{n} c_{i}\left|a_{i}-f_{i}\right|=\sum_{i=1}^{n} c_{i}\left(a_{i}-f_{i}\right) .
$$

(iv) For $g^{c}=\max _{i} a_{i}$, then

$$
M C C_{2}=\sum_{i=1}^{n} c_{i}\left|a_{i}-g_{i}\right|=\sum_{i=1}^{n} c_{i}\left(g_{i}-a_{i}\right) .
$$

Based on Eqs. (22) and (24), it is

$$
M C C_{1}-M C C_{3}=\sum_{i=1}^{n} c_{i}\left(a_{i}-b_{i}\right)-\sum_{i=1}^{n} c_{i}\left(a_{i}-f_{i}\right)=\sum_{i=1}^{n} c_{i}\left(f_{i}-b_{i}\right) .
$$

The feasible solutions $\left\{b_{1}, b_{2}, \ldots, b_{n}, b^{c}\right\}$ and $\left\{f_{1}, f_{2}, \ldots, f_{n}, f^{c}\right\}$ satisfy the condition $\left|b_{1}-b^{c}\right| \leq \varepsilon$ and $\left|f_{1}-f^{c}\right| \leq \varepsilon(i=1,2, \ldots, n)$ of model $P_{1}$, then $f_{i} \geq f^{c}-\varepsilon$ and $b_{i} \leq$ $b^{c}+\varepsilon($ for all $i=1,2, \ldots, n)$, hence

$$
M C C_{1}-M C C_{3}=\sum_{i=1}^{n} c_{i}\left(f_{i}-b_{i}\right) \geq \sum_{i=1}^{n} c_{i}\left(f^{c}-b^{c}-2 \varepsilon\right) .
$$

When $\varepsilon$ tends to $0, \sum_{i=1}^{n} c_{i}\left(f^{c}-b^{c}-2 \varepsilon\right)$ tends to $\sum_{i=1}^{n} c_{i}\left(f^{c}-b^{c}\right)>0$ and therefore it is

$$
M C C_{1}-M C C_{3}>0
$$

Thus, $M C C_{1}>M C C_{3}$.

Based on Eqs. (23) and (25), we have

$$
M C C_{2}-M C C_{4}=\sum_{i=1}^{n} c_{i}\left(e_{i}-a_{i}\right)-\sum_{i=1}^{n} c_{i}\left(g_{i}-a_{i}\right)=\sum_{i=1}^{n} c_{i}\left(e_{i}-g_{i}\right) .
$$

The feasible solutions $\left\{e_{1}, e_{2}, \ldots, e_{n}, e^{c}\right\}$ and $\left\{g_{1}, g_{2}, \ldots, g_{n}, g^{c}\right\}$ satisfy the condition $\left|e_{1}-e^{c}\right| \leq \varepsilon$ and $\left|g_{1}-g^{c}\right| \leq \varepsilon(i=1,2, \ldots, n)$ of model $P_{1}$, then $e_{i} \geq e^{c}-\varepsilon$ and $g_{i} \leq$ $g^{c}+\varepsilon($ for all $i=1,2, \ldots, n)$, hence

$$
M C C_{2}-M C C_{4}=\sum_{i=1}^{n} c_{i}\left(e_{i}-g_{i}\right) \geq \sum_{i=1}^{n} c_{i}\left(e_{i}-g_{i}-2 \varepsilon\right) .
$$

Taking limits when $\varepsilon$ tends to 0 , we would get that

$$
M C C_{2}-M C C_{4} \geq \sum_{i=1}^{n} c_{i}\left(e_{i}-g_{i}\right)>0 .
$$

Thus, $M C C_{2}>M C C_{4}$.

Accordingly, it is clear that $\left[0, \min _{i} a_{i}\right) \cup\left(\max _{i} a_{i}, 1\right]$ is excluded from the range of the optimal collective opinion $o^{c}$ of model $P_{l}$. Therefore, it is concluded that $o^{c} \in$ $\left[\min _{i} a_{i}, \max _{i} a_{i}\right]$.

This completes the proof of Lemma 1.

Theorem 2: Let $\left\{p_{1}, p_{2}, \ldots, p_{n}, p^{c}\right\}$ denote the optimal solution of model $P_{6}$. Then, it is: 


$$
p^{c}=\left\{\begin{array}{l}
\max _{i} p_{i}, 2 \sum_{i=1}^{n} c_{i} a_{i}-\left(\min _{i} a_{i}+\max _{i} a_{i}\right) \sum_{i=1}^{n} c_{i}<0 \\
\min _{i} p_{i}, 2 \sum_{i=1}^{n} c_{i} a_{i}-\left(\min _{i} a_{i}+\max _{i} a_{i}\right) \sum_{i=1}^{n} c_{i}>0
\end{array} .\right.
$$

\section{Proof:}

The process of proving Theorem 2 is divided into two steps.

Step 1: Proof $p^{c} \in\left[\min _{i} a_{i}, \max _{i} a_{i}\right]$.

The aim of solving model $P_{l}$ is to obtain the optimal solution under the fixed decision rule, while solving model $P_{6}$ aims at obtaining the optimal adjusted collective opinion that maximizes the MCC, which equals to finding the optimal decision rule that maximizes the MCC based on the Eq. (5).

The MCC obtained with model $P_{1}$ with this optimal decision rule is the largest of all MCC obtained with model $P_{1}$ with any decision rule. Obviously, Lemma 1 still applies to model $P_{6}$ because the optimal solution $\left\{p_{1}, p_{2}, \ldots, p_{n}, p^{c}\right\}$ of model $P_{6}$ is also the optimal solution of model $P_{1}$ with this optimal decision rule. Hence, it is concluded that $p^{c} \in\left[\min _{i} a_{i}, \max _{i} a_{i}\right]$.

Step 2: Proof $p^{c} \rightarrow \min _{i} p_{i}$ or $\max _{i} p_{i}$

Let $\left\{h_{1}, h_{2}, \ldots, h_{n}, h^{c}\right\},\left\{f_{1}, f_{2}, \ldots, f_{n}, f^{c}\right\}$, and $\left\{g_{1}, g_{2}, \ldots, g_{n}, g^{c}\right\}$ denote feasible solutions of model $P_{6}$, where $h^{c} \in\left(\min _{i} a_{i}, \max _{i} a_{i}\right), f^{c}=\min _{i} a_{i}$ and $g^{c}=\max _{i} a_{i}$ and $\varepsilon$ is a small enough number.

(i) For $h^{c} \in\left(\min _{i} a_{i}, \max _{i} a_{i}\right)$, it is

$$
M C C_{1}=\sum_{i=1}^{n} c_{i}\left|a_{i}-h_{i}\right|=\sum_{i: h_{i} \geq a_{i}} c_{i}\left(h_{i}-a_{i}\right)+\sum_{i: h_{i}<a_{i}} c_{i}\left(a_{i}-h_{i}\right) .
$$

(ii) For $f^{c}=\min _{i} a_{i}$, it is

$$
M C C_{2}=\sum_{i=1}^{n} c_{i}\left|a_{i}-f_{i}\right|=\sum_{i=1}^{n} c_{i}\left(a_{i}-f_{i}\right)=\sum_{i: h_{i} \geq a_{i}} c_{i}\left(a_{i}-f_{i}\right)+\sum_{i: h_{i}<a_{i}} c_{i}\left(a_{i}-f_{i}\right) .
$$

(iii) For $g^{c}=\max _{i} a_{i}$, it is

$$
M C C_{3}=\sum_{i=1}^{n} c_{i}\left|a_{i}-g_{i}\right|=\sum_{i=1}^{n} c_{i}\left(g_{i}-a_{i}\right)=\sum_{i: h_{i} \geq a_{i}} c_{i}\left(g_{i}-a_{i}\right)+\sum_{i: h_{i}<a_{i}} c_{i}\left(g_{i}-\right.
$$

Based on Eqs. (33) and (34), it is

$$
\begin{aligned}
M C C_{2}-M_{1} C_{1} & =\sum_{i: h_{i} \geq a_{i}} c_{i}\left(2 a_{i}-f_{i}-h_{i}\right)+\sum_{i: h_{i}<a_{i}} c_{i}\left(h_{i}-f_{i}\right) \\
& \geq \sum_{i: h_{i} \geq a_{i}} c_{i}\left(2 h_{i}-f_{i}-h_{i}\right)+\sum_{i: h_{i}<a_{i}} c_{i}\left(h_{i}-f_{i}\right) \\
& \geq \sum_{i: h_{i} \geq a_{i}} c_{i}\left(h_{i}-f_{i}\right)+\sum_{i: h_{i}<a_{i}} c_{i}\left(h_{i}-f_{i}\right) \\
& \geq \sum_{i=1}^{n} c_{i}\left(h_{i}-f_{i}\right) .
\end{aligned}
$$

The feasible solutions $\left\{h_{1}, h_{2}, \ldots, h_{n}, h^{c}\right\}$ and $\left\{f_{1}, f_{2}, \ldots, f_{n}, f^{c}\right\}$ satisfy the conditions $\left|h_{1}-h^{c}\right| \leq \varepsilon$ and $\left|f_{1}-f^{c}\right| \leq \varepsilon(i=1,2, \ldots, n)$ of model $P_{6}$, then $h_{i} \geq h^{c}-\varepsilon$ and $f_{i} \leq$ 
$f^{c}+\varepsilon$ (for all $i=1,2, \ldots, n$ ). Taking limits when $\varepsilon$ tends to 0 , we would get that

$$
M C C_{2}-M C C_{1} \geq \sum_{i=1}^{n} c_{i}\left(h^{c}-f^{c}\right)>0 .
$$

Based on Eqs. (33) and (35), it is

$$
\begin{aligned}
M C C_{3}-M C C_{1}=\sum_{i: h_{i} \geq a_{i}} c_{i}\left(g_{i}-h_{i}\right)+\sum_{i: h_{i}<a_{i}} c_{i}\left(g_{i}+h_{i}-2 a_{i}\right) \\
\quad \geq \sum_{i: h_{i} \geq a_{i}} c_{i}\left(g_{i}-h_{i}\right)+\sum_{i: h_{i}<a_{i}} c_{i}\left(g_{i}+h_{i}-2 h_{i}\right) \\
\geq \sum_{i: h_{i} \geq a_{i}} c_{i}\left(g_{i}-h_{i}\right)+\sum_{i: h_{i}<a_{i}} c_{i}\left(g_{i}-h_{i}\right) \\
\geq \sum_{i=1}^{n} c_{i}\left(g_{i}-h_{i}\right) .
\end{aligned}
$$

The feasible solutions $\left\{h_{1}, h_{2}, \ldots, h_{n}, h^{c}\right\}$ and $\left\{g_{1}, g_{2}, \ldots, g_{n}, g^{c}\right\}$ satisfy the conditions $\left|h_{1}-h^{c}\right| \leq \varepsilon$ and $\left|g_{1}-g^{c}\right| \leq \varepsilon(i=1,2, \ldots, n)$ of model $P_{6}$, then $h_{i} \leq h^{c}+\varepsilon$ and $g_{i} \geq$ $g^{c}-\varepsilon$ (for all $\left.i=1,2, \ldots, n\right)$. Simlarly, it would be

$$
M C C_{3}-M C C_{1} \geq \sum_{i=1}^{n} C_{i}\left(g^{c}-h^{c}\right)>0 .
$$

From Eqs. (37) and (39), it can be concluded that $M C C_{2}>M C C_{1}$ and $M C C_{3}>M C C_{1}$ when $\varepsilon$ is a small enough number, i.e. the closer $p^{c}$ is to $\min _{i} a_{i}$ or $\max _{i} a_{i}$, the larger the MCC is. Because $p^{c} \in\left[\min _{i} p_{i}, \max _{i} p_{i}\right]$, this conclusion are guaranteed in the limit case, i.e. $p^{c}=\min _{i} p_{i}$ when $p^{c} \rightarrow \min _{i} a_{i}$ and $p^{c}=\max _{i} p_{i}$ when $p^{c} \rightarrow \max _{i} a_{i}$. Therefore, we have

$$
p^{c}=\left\{\begin{array}{l}
\max _{i} p_{i}, M C C_{3}>M C C_{2} \\
\min _{i} p_{i}, M C C_{3}<M C C_{2}
\end{array}\right.
$$

Taking limits when $\varepsilon$ tends to 0 , we would get that

$$
p^{c}=\left\{\begin{array}{l}
\max _{i} p_{i}, 2 \sum_{i=1}^{n} c_{i} a_{i}-\left(\min _{i} a_{i}+\max _{i} a_{i}\right) \sum_{i=1}^{n} c_{i}<0 \\
\min _{i} p_{i}, 2 \sum_{i=1}^{n} c_{i} a_{i}-\left(\min _{i} a_{i}+\max _{i} a_{i}\right) \sum_{i=1}^{n} c_{i}>0
\end{array}\right.
$$

This completes the proof of Theorem 2.

Based on the Theorem 2, the decision rule that maximizes the MCC of model $P_{6}$ is

$$
\overline{a^{c}}=F_{O W A}\left(\overline{a_{1}}, \overline{a_{2}}, \ldots, \overline{a_{n}}\right),
$$

with the following associated decision weight vector

$$
\text { OWA_max }=\left\{\begin{array}{l}
(1,0, \ldots, 0)^{T}, 2 \sum_{i=1}^{n} c_{i} a_{i}-\left(\min _{i} a_{i}+\max _{i} a_{i}\right) \sum_{i=1}^{n} c_{i}<0 \\
(0, \ldots, 0,1)^{T}, 2 \sum_{i=1}^{n} c_{i} a_{i}-\left(\min _{i} a_{i}+\max _{i} a_{i}\right) \sum_{i=1}^{n} c_{i}>0
\end{array} .\right.
$$

\section{Simulation analysis}

In this section, we further reveal the impact of non-cooperative behaviors and decision rules on MCC via Simulation experiments I, II and III. In the minimum-cost consensus model, $c_{i}$ denotes the cost of moving $d_{i}$ 's opinion 1 unit, and in this study we argue that it can 
represent the non-cooperative behavior coefficient of decision maker $d_{i}$ because larger $c_{i}$ values means less cooperation to reach consensus. Thus, $C=\left\{c_{1}, c_{2}, \ldots, c_{n}\right\}$ is considered to be the set of non-cooperative behavior coefficients. Meanwhile, we argue that the decision rules consist of the aggregation function and the associated decision weight.

\subsection{Simulation experiment I: Impact of non-cooperative behaviors on MCC}

The basic idea of Simulation experiment I is to randomly generate original individual opinions and values of $c_{i}(i=1,2, \ldots, n)$. Particularly, $c_{i}$ is randomly generated based on the normal distribution $N\left(\mu_{c}, \sigma_{c}^{2}\right)$ subject to the constraint $c_{i}>0$, where $\mu_{c}$ and $\sigma_{c}^{2}$ approximately measure the expectation and variance of $d_{i}$ 's non-cooperative behavior coefficient, respectively. Thus, we study the impact of non-cooperative behaviors on MCC through $\mu_{c}$ and $\sigma_{c}^{2}$. Simulation experiment I can be formally presented as follows.

\section{Simulation experiment I:}

Input: The number of decision makers $n$, the expectation of $d_{i}$ 's non-cooperative behavior coefficient $\mu_{c}$, the variance of $d_{i}$ 's non-cooperative behavior coefficient $\sigma_{c}^{2}$, the established threshold value $\varepsilon$ and decision weight $w=\left(w_{1}, w_{2}, \ldots, w_{n}\right)^{T}$.

Output: The minimum consensus cost, MCC.

Step 1: Let $A=\left\{a_{1}, a_{2}, \ldots, a_{n}\right\}$ be the original individual opinion set in model $P_{2}$, where $a_{i}(i=1,2, \ldots, n)$ is randomly selected using the uniform distribution on $[0,1]$.

Step 2: Let $C=\left\{c_{1}, c_{2}, \ldots, c_{n}\right\}$ be the set of non-cooperative behavior coefficients in model $P_{2}$, where $c_{i}(i=1,2, \ldots, n)$ is randomly generated based on $N\left(\mu_{c}, \sigma_{c}^{2}\right)$ subject to the constraint $c_{i}>0$.

Step 3: Compute the MCC based on model $P_{2}$.

Without loss of generality, in the simulation we consider two decision rules:

$$
O W A_{1}=(1 / n, \ldots, 1 / n)^{T} \text { and } O W A_{2}=(0,1 /(n-2), \ldots, 1 /(n-2), 0)^{T} \text {. }
$$

We set different values for $n, \varepsilon, \mu_{c}, \sigma_{c}^{2}$ and run Simulation experiment I 10,000 times to calculate the average of the MCC values, which is shown in Figs. 2 and 3. 
(a) $\mathbf{n}=5, \epsilon=0.10, O W A_{1}$

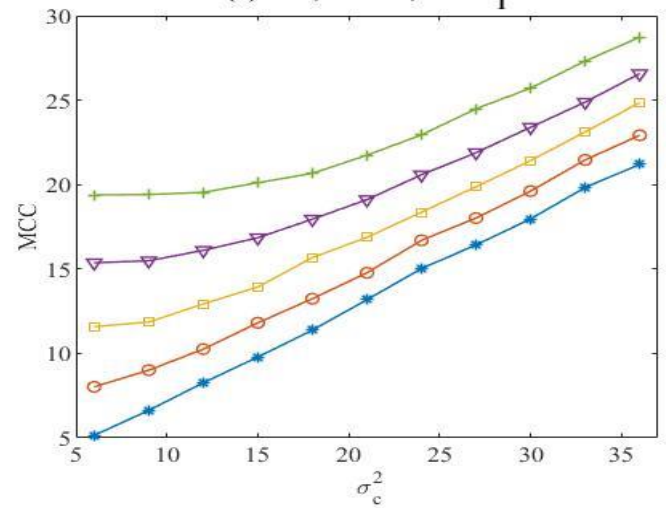

(c) $\mathbf{n}=5, \epsilon=0.05, \mathrm{OWA}$

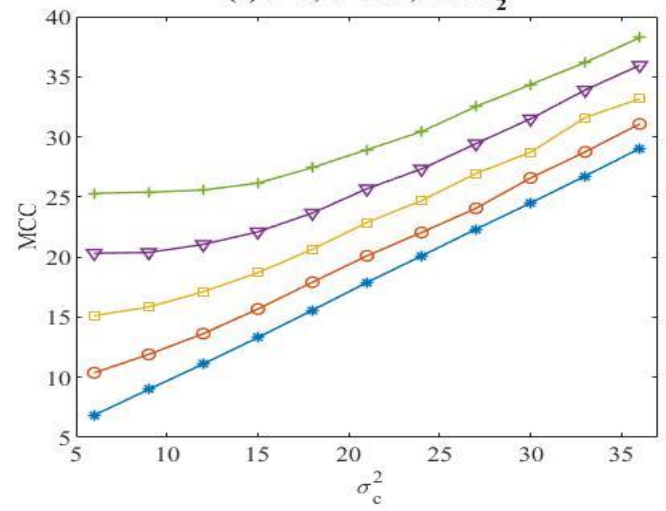

(b) $\mathbf{n}=7, \epsilon=0.10$, OWA

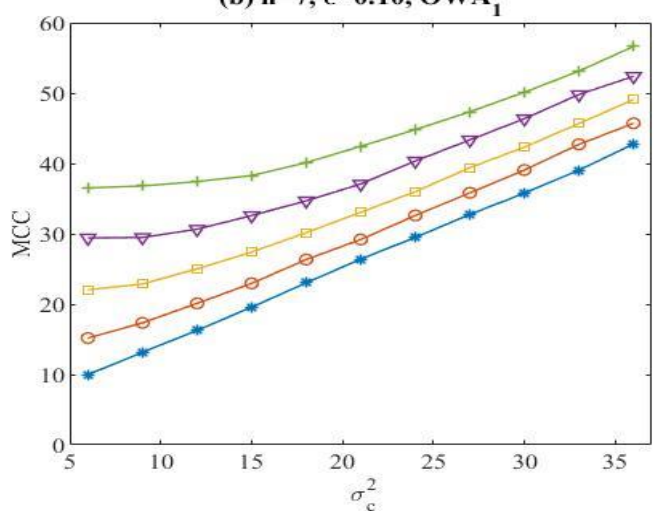

(d) $\mathbf{n}=7, \epsilon=0.05$, OWA 2

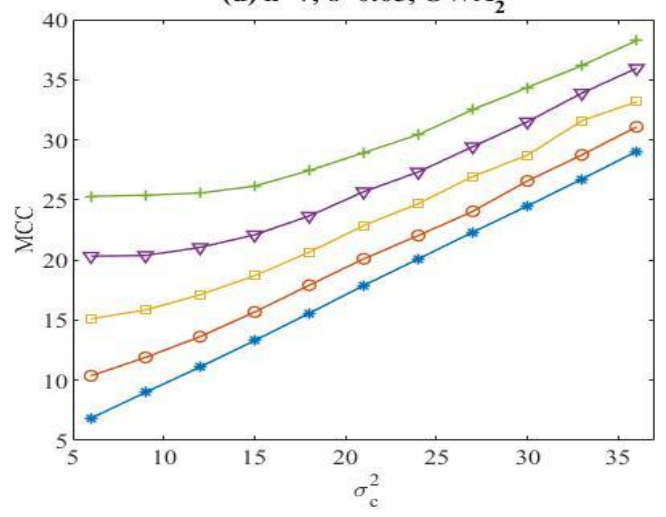

$\longrightarrow \mu_{\mathrm{c}}=5 \longrightarrow \mu_{\mathrm{c}}=10 \longrightarrow \mu_{\mathrm{c}}=15 \longrightarrow \mu_{\mathrm{c}}=20 \div \mu_{\mathrm{c}}=25$

Fig. 2 Effects of $\mu_{c}$ and $\sigma_{c}^{2}$ on the MCC under different parameters 

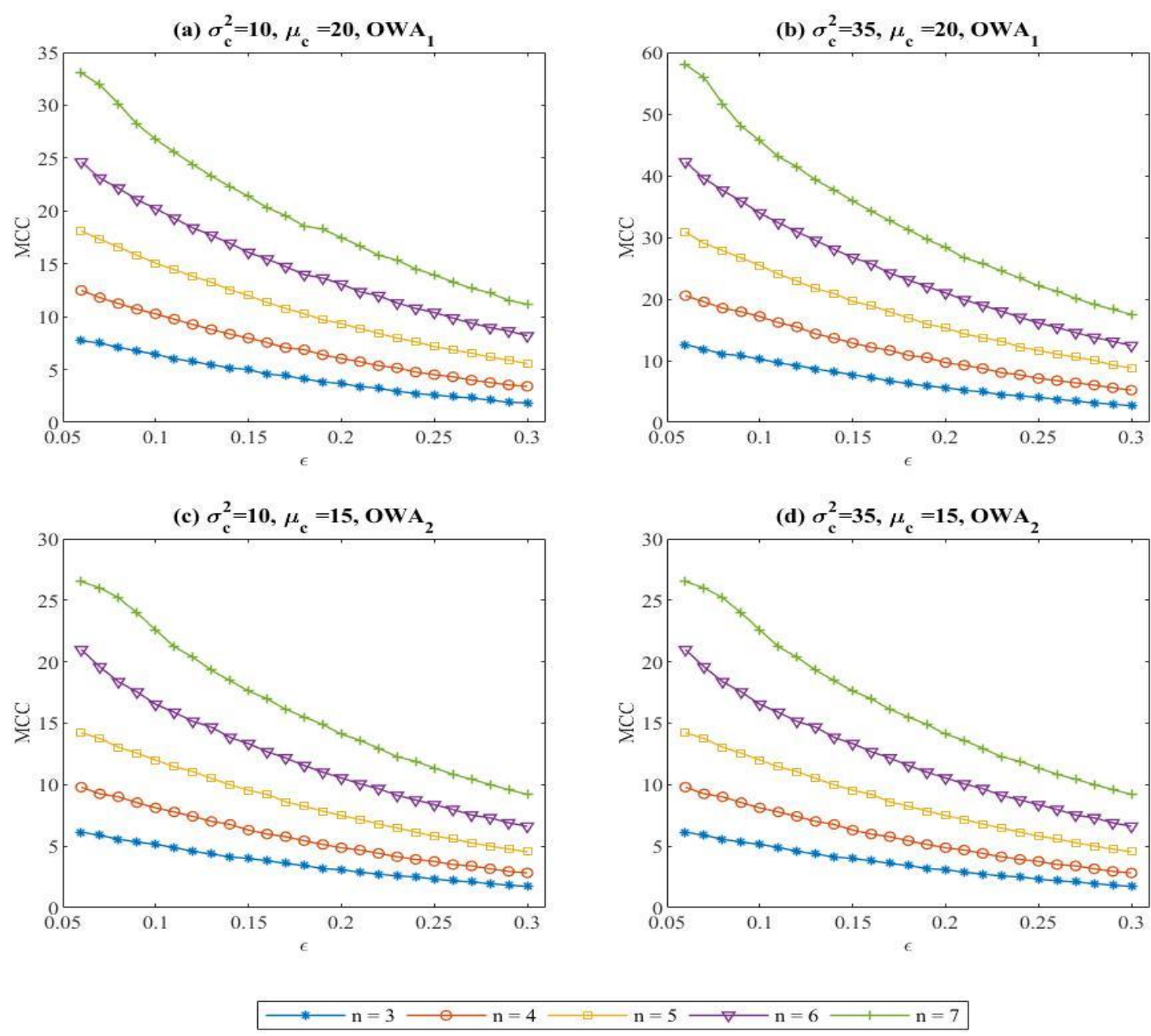

Fig. 3 Effects of $\varepsilon$ and $n$ on the MCC under different parameters

From Figs. 2 and 3, we draw the following observations:

(1) Fig. 2 shows that the MCC increases as $\mu_{c}$ and $\sigma_{c}^{2}$ increase under different parameters. This implies that the non-cooperative behaviors in the group, defined by $\mu_{c}$ and $\sigma_{c}^{2}$, strongly increase the MCC, which translates in an increase in the difficulty when reaching consensus in GDM.

(2) Fig. 3 shows that the MCC increases as $n$ increases under different parameters, being this increment more significant the smaller $\varepsilon$ values or the larger $\sigma_{c}^{2}$ values are, respectively. This agrees with the real increase of difficulty in reaching consensus the larger the group experts. Meanwhile, the number of decision makers will have a stronger impact on the consensus reaching in GDM when decision makers are less tolerant to inconsistent views, defined by $\varepsilon$, or the larger the non-cooperative behaviors in the group are, than otherwise.

\subsection{Simulation experiment II: The joint impact of non-cooperative behaviors and} decisions rules on MCC

Let $C=\left\{c_{1}, c_{2}, \ldots, c_{n}\right\}$ be the set of non-cooperative behavior coefficients, defined by unit cost; $S(C)=\sum_{i=1}^{n} c_{i}$ the sum of the non-cooperative behavior coefficients; and $D(C)=$ 
$\left(\sum_{i=1}^{n}\left[c_{i}-S(C) / n\right]^{2}\right) / n$ the variance of the non-cooperative behavior coefficients. Let $w_{i}=\overline{w_{i}} / \sum_{j=1}^{n} \overline{w_{j}}(i=1,2, \ldots, n)$ and $\overline{w_{i}}$ be randomly generated using normal distribution $N\left(0.2, \sigma_{w}^{2}\right)$ subject to the constraint $\overline{w_{i}}>0$. Then, $\sigma_{w}^{2}$ approximately measures the variance of decision weights.

The basic idea of Simulation experiment II is to randomly generate original individual opinions, and the values of $w_{i}(i=1,2, \ldots, n)$. Then, we investigate the joint impact of non-cooperative behaviors and decisions rules on MCC through $C$ and $\sigma_{w}^{2}$. Simulation experiment II can be formally presented as follows.

\section{Simulation experiment II:}

Input: The number of decision makers $n$, the variance of decision weights $\sigma_{w}^{2}$, the established threshold value $\varepsilon$ and the non-cooperative behavior coefficient set $C=$ $\left\{c_{1}, c_{2}, \ldots, c_{n}\right\}$.

Output: The minimum consensus cost, MCC.

Step 1: Let $A=\left\{a_{1}, a_{2}, \ldots, a_{n}\right\}$ be the original individual opinion set of model $P_{2}$ (or model $\left.P_{3}\right)$. where $a_{i}(i=1,2, \ldots, n)$ is randomly selected using the uniform distribution on $[0,1]$..

Step 2: Let $W=\left(w_{1}, w_{2}, \ldots, w_{n}\right)^{T}$ be decision weight of model $P_{2}$ (or model $P_{3}$ ), where $w_{i}=\overline{w_{i}} / \sum_{j=1}^{n} \overline{w_{j}}(i=1,2, \ldots, n)$, and $\overline{w_{i}}$ is randomly generated using normal distribution $N\left(0.2, \sigma_{w}^{2}\right)$ subject to the constraint $\overline{w_{i}}>0$.

Step 3: Compute the MCC based on model $P_{2}$ (or model $P_{3}$ ).

In order to reflect the impact of $D(C)$ on $\mathrm{MCC}$, we consider eight non-cooperative behavior coefficients sets:

$$
\begin{aligned}
& C_{1}=\{3,3,3,3,3\}, \quad C_{2}=\{5,4,3,2,1\}, \quad C_{3}=\{1,2,7,3,2\}, \quad C_{4}=\{8,5,2,0,0\}, \\
& C_{5}=\{10,2,1,1,1\}, \quad C_{6}=\{1,2,3,4,5\}, \quad C_{7}=\{7,3,2,1,2\}, \quad C_{8}=\{0,0,8,5,2\},
\end{aligned}
$$

with same sum $S\left(C_{k}\right)=15(k=1,2, \ldots, 8)$ but different variance values:

$$
\begin{gathered}
D\left(C_{1}\right)=0, D\left(C_{2}\right)=2, D\left(C_{3}\right)=4.4, D\left(C_{4}\right)=9.6, \\
D\left(C_{5}\right)=12.4, D\left(C_{6}\right)=2, D\left(C_{7}\right)=4.4, D\left(C_{8}\right)=9.6 .
\end{gathered}
$$

We set different $\sigma_{w}^{2}$ values and run Simulation experiment II 10,000 times to calculate the average of the MCC values, which are shown in Figs. 4 and 5. 

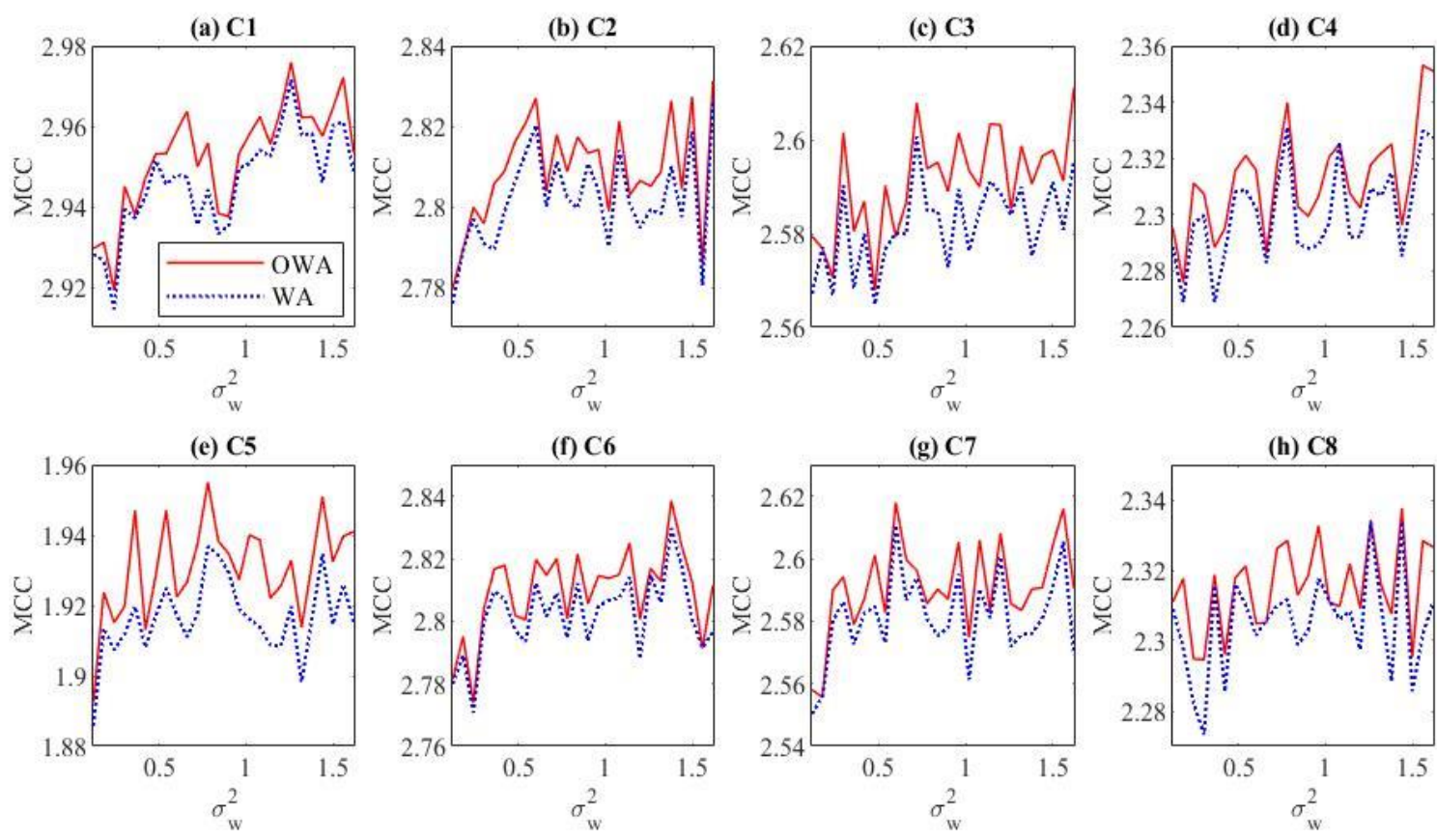

Fig. 4 The difference of models $P_{2}$ (OWA) and $P_{3}$ (WA) on MCC with $n=5$ and $\varepsilon=0.05$
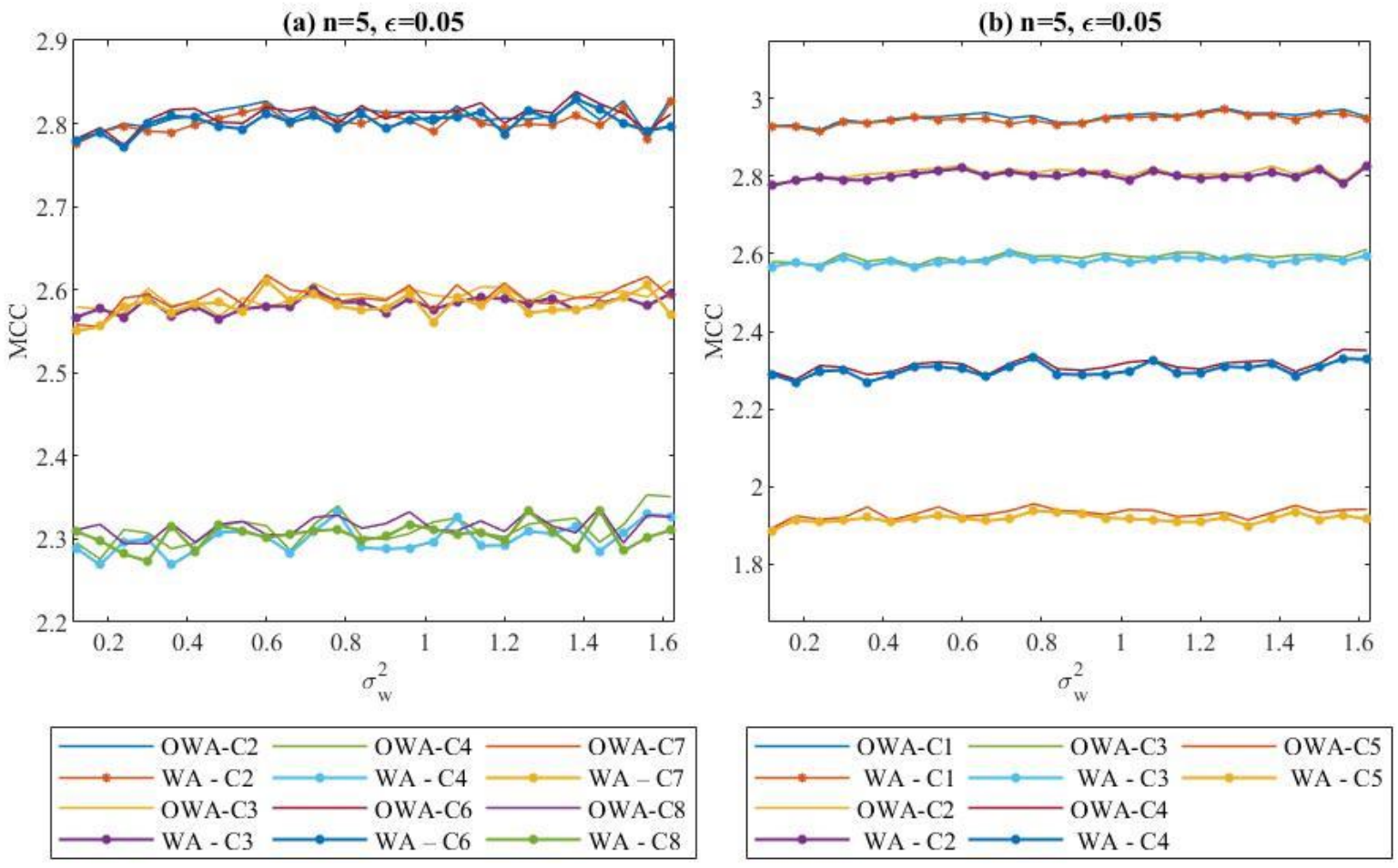

Fig. 5 The joint impact of $\sigma_{w}^{2}$ and $D(C)$ on MCC

From Figs. 4 and 5, we draw the following observations:

(1) Fig. 4 shows that the MCC obtained with model $P_{2}$ is larger than the one obtained with model $P_{3}$ under different parameters, which means that it is more difficult to reach consensus using OWA than using WA under the same decision weight.

(2) In Fig. 5 (a), $\mathrm{S}\left(C_{k}\right)=15(k=1,2,3,4,5), D\left(C_{1}\right)<D\left(C_{2}\right)<D\left(C_{3}\right)<D\left(C_{4}\right)<D\left(C_{5}\right)$; 
and in Fig. 5 (b), $S\left(C_{k}\right)=15 \quad(k=2,3,4,6,8), \quad D\left(C_{2}\right)=D\left(C_{6}\right)<D\left(C_{3}\right)=D\left(C_{7}\right)<$ $D\left(C_{4}\right)=D\left(C_{8}\right)$. Fig. 5 (a) shows that the MCC, obtained with model $P_{2}$ and $P_{3}$, slightly fluctuate around the same fixed value as $\sigma_{w}^{2}$ increases under the same $D(C)$ and $S(C)$ values. Fig. 5 (b) shows that larger $D(C)$ values lead to lower MCC under the same $S(C)$ values. This implies that decision rules, defined by decision weights and the aggregation functions, are insensitive to the MCC, while $C$, defined by $D(C)$ and $S(C)$, is a main determinant in influencing the MCC.

\subsection{Simulation experiment III: The impact of the consensus within the established target on MCC}

This section illustrates the relation between MCC and building a consensus opinion $\overline{a^{c}}$ within a desired range $[a, b]$.We firstly consider the following minimum-cost consensus model $P_{7}$,

$$
\begin{gathered}
\min _{\overline{a_{i}}} \sum_{i=1}^{n} c_{i}\left|a_{i}-\overline{a_{i}}\right| \\
\overline{a^{c}}=F_{O W A}\left(\overline{a_{1}}, \overline{a_{2}}, \ldots, \overline{a_{n}}\right), \\
\left|\overline{a_{i}}-\overline{a^{c}}\right| \leq \varepsilon, i=1,2, \ldots, n \\
a \leq \overline{a^{c}} \leq b
\end{gathered}
$$

The basic idea of Simulation experiment III is to randomly generate original individual opinions, and the values of $c_{i}(i=1,2, \ldots, n)$ to investigate the effect of the consensus, within the established target, on MCC through $\mu_{c}, \sigma_{c}^{2}$, and $[a, b]$. Simulation experiment III can be formally presented as follows.

\section{Simulation experiment III:}

Input: The number of decision makers $n$, the expectation of $d_{i}$ 's non-cooperative behavior coefficient $\mu_{c}$, the variance of $d_{i}$ 's non-cooperative behavior coefficient $\sigma_{c}^{2}$, the established threshold value $\varepsilon$, decision weight $w=\left(w_{1}, w_{2}, \ldots, w_{n}\right)^{T}$ and the established collective solution target range $[a, b]$.

Output: The minimum consensus cost, MCC.

Step 1: Let $A=\left\{a_{1}, a_{2}, \ldots, a_{n}\right\}$ be the original individual opinion set in model $P_{7}$, where $a_{i}(i=1,2, \ldots, n)$ is randomly selected using the uniform distribution on $[0,1]$.

Step 2: Let $C=\left\{c_{1}, c_{2}, \ldots, c_{n}\right\}$ be the set of non-cooperative behavior coefficients in model $P_{7}$, where $c_{i}(i=1,2, \ldots, n)$ is randomly generated using normal distribution $N\left(\mu_{c}, \sigma_{c}^{2}\right)$ subject to the constraint $c_{i}>0$.

Step 3: Compute the MCC based on model $P_{7}$.

Without loss of generality, in the simulation we consider two decision rules as follow:

$$
O W A_{1}=(1 / n, \ldots, 1 / n)^{T} \text { and } O W A_{2}=(0,1 /(n-2), \ldots, 1 /(n-2), 0)^{T} \text {. }
$$

We set different values for $n, \varepsilon, \mu_{c}, \sigma_{c}^{2},[a, b]$ and run Simulation experiment III 10,000 
times to calculate the average of the MCC values, which is shown in Figs. 6 and 7.
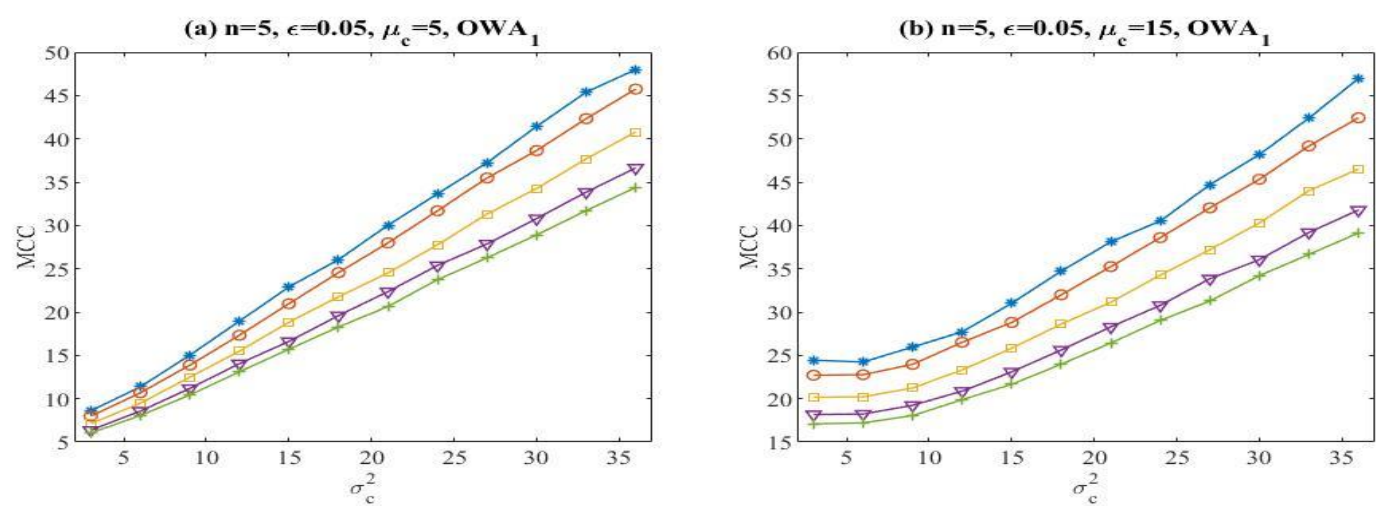

(c) $\mathrm{n}=5, \epsilon=0.05, \mu_{\mathrm{c}}=5$, OWA

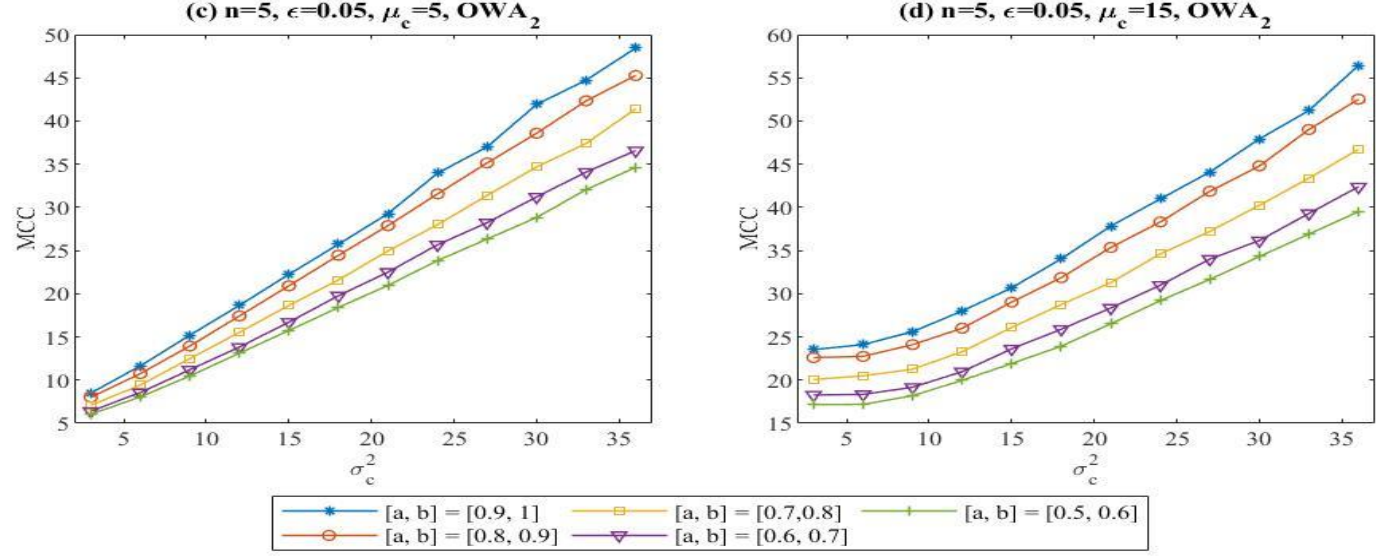

Fig. 6 Effects of $[a, b]$ and $\sigma_{c}^{2}$ on the MCC under different parameters
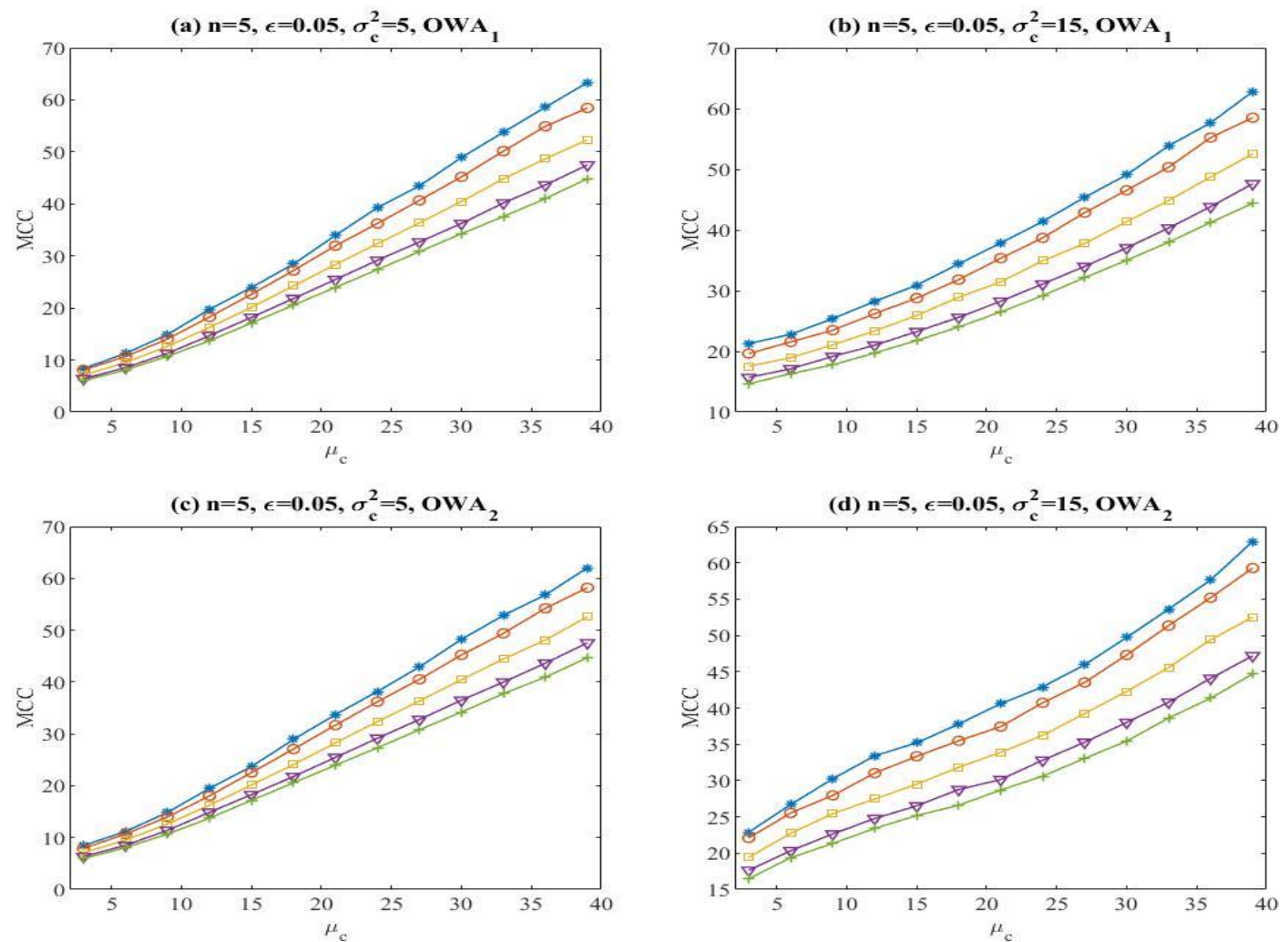

$\begin{aligned} & \square {[\mathrm{a}, \mathrm{b}]=[0.9,1] } \\ & \square\mathrm{a}, \mathrm{b}]=[0.8,0.9] \longrightarrow[\mathrm{a}, \mathrm{b}]=[0.7,0.8] \longrightarrow \\ & \square \quad[\mathrm{a}, \mathrm{b}]=[0.6,0.7]\end{aligned}$ 
Fig. 7 Effects of $[a, b]$ and $\mu_{c}$ on the MCC under different parameters

From Figs. 6 and 7, we draw the following observations:

(1) Likewise to the observations previously drawn from Figs. 2 and 3, Figs. 6 and 7 also show that the MCC increases as $\mu_{c}$ and $\sigma_{c}^{2}$ increase under different parameters.

(2) Figs. 6 and 7 show that the MCC increases when the established collective solution target range moves up from $[0.5,0.6]$ to $[0.9,1]$ through $[0.6,0.7],[0.7,0.8],[0.8,0.9]$, which means that it is more difficult to achieve an extreme collective consensus opinion. Meanwhile, this inference is more obvious under larger non-cooperative behaviors in the group, defined by $\mu_{c}$ and $\sigma_{c}^{2}$.

\section{Conclusion}

This paper studied the impact of decision rules and non-cooperative behaviors on MCC in GDM. Theoretically, the decision rule that minimizes or maximizes MCC were derived. Furthermore, the joint impact of non-cooperative behaviors and decision rules on MCC were analyzed via simulation experiments.

In general, a preference adjustment has associated a cost and the consensus reaching resources are limited, which implies that the results obtained in this paper can provide new perspectives to understand how decision rules and non-cooperative behaviors influence the consensus cost.

Meanwhile, social network and opinion dynamics are becoming new tools to model CRP (Dong et al., 2018a; Gong et al., 2020), and we argue that it will be promising to investigate the consensus with minimum cost in social network and opinion dynamics contexts. In particular, it is very necessary to investigate the impact of decision rules and non-cooperative behaviors on MCC in social network and opinion dynamics contexts.

\section{References}

Akram, M., \& Arshad, M. (2018). A novel trapezoidal bipolar fuzzy topsis method for group decision-making. Group Decision and Negotiation, 28(3), 565-584.

Akram, M., Adeel, A., \& Alcantud, J. C. R. (2019a).Group decision-making methods based on hesitant N-soft sets. Expert Systems with Applications, 115, 95-105.

Akram, M., Ali, G., \& Alcantud, J. C. R. (2019b). New decision-making hybrid model: intuitionistic fuzzy N-soft rough sets. Soft Computing, 23(20), 9853-9868.

Akram, M., Ilyas, F., \& Garg, H. (2019c). Multi-criteria group decisionmaking based on ELECTRE I method in Pythagorean fuzzy information. Soft Computing, in press, DOI: 10.1007/s00500-019-04105-0.

Ben-Arieh, D., \& Easton, T. (2007). Multi-criteria group consensus under linear cost opinion elasticity. Decision Support Systems, 43(3), 713-721.

Ben-Arieh, D., Easton, T., \& Evans, B. (2008). Minimum cost consensus with quadratic cost functions. IEEE Transactions on Systems, Man and Cybernetics-Part A: Systems and Humans, 39(1), 210-217.

Cheng, D., Zhou, Z. L., Cheng, F. X., Zhou, Y. F., \& Xie, Y. J. (2018). Modeling the minimum cost consensus 
problem in an asymmetric costs context. European Journal of Operational Research, 270, 1122-1137.

Chiclana, F., Tapia García, J. M., Del Moral, M. J., \& Herrera-Viedma, E. (2013). A statistical comparative study of different similarity measures of consensus in group decision making. Information Sciences, 221,110-123.

Dong, Y. C., \& Xu, J. P. (2016). Consensus building in group decision making: Searching the consensus path with minimum adjustments. Springer-Verlag.

Dong, Y. C., Li, C. C., Xu, Y. F., \& Gu, X. (2015). Consensus-based group decision making under multi-granular unbalanced 2-tuple linguistic preference relations. Group Decision and Negotiation, 24, 217-242

Dong, Y. C., Xu, Y .F., Li, H. Y., \& Feng, B. (2010). The OWA-based consensus operator under linguistic representation models using position indexes. European Journal of Operational Research, 203(2), 455-463

Dong, Y. C., Zha, Q. B., Zhang, H. J., Kou, G., Fujita, H., Chiclana, F., \& Herrera-Viedma, E. (2018a). Consensus reaching in social network group decision making: Research paradigms and challenges. Knowledge-Based Systems, 162, 3-13.

Dong, Y. C., Zhang, H. J., \& Herrera-Viedma, E. (2016). Integrating experts' weights generated dynamically into the consensus reaching process and its application in managing non-cooperative behaviors. Decision Support Systems, 84, 1-15.

Dong, Y. C., Zhao, S. H., Zhang, H. J., Chiclana, F., \& Herrera-Viedma, E. (2018b). A self-management mechanism for non-cooperative behaviors in large-scale group consensus reaching processes. IEEE Tran-sactions on Fuzzy Systems, 26(6), 3276-3288.

Dong, Y.C., Liu, Y. T., Liang, H. M., Chiclana, F., \& Herrera-Viedma, E. (2017). Strategic weight manipulation in multiple attribute decision making. Omega, 75, 154-164.

Fedrizzi, K. M. (1988). A 'soft' measure of consensus in the setting of partial (fuzzy) preferences. European Journal of Operational Research, 34(3), 316-325.

Gong, Z. W., Guo, W. W., Herrera-Viedma, E., Gong, Z. J., \& Wei, G. (2019). Consistency and consensus modeling of linear uncertain preference relations, European Journal of Operational Research, in press, DOI: 10.1016/j.ejor.2019.10.035.

Gong, Z. W., Wang, H., Guo, W. W., Gong, Z. J., \& Wei, G. (2020). Measuring trust in social networks based on linear uncertainty theory. Information Sciences, 508, 154-172.

Gong, Z. W., Xu, C., Chiclana, F., \& Xu, X. X. (2017). Consensus measure with multi-stage fluctuation utility based on china's urban demolition negotiation. Group Decision and Negotiation, 26(2), 379-407

Gong, Z. W., Xu, X. X., Lu, F. L., Li, L. S., \& Xu, C. (2015a). On consensus models with utility preferences and limited budget. Applied Soft Computing, 35, 840-849.

Gong, Z. W., Zhang, H. H., Forrest, J., Li, L., \& Xu, X. (2015b). Two consensus models based on the minimum cost and maximum return regarding either all individuals or one individual. European Journal of Operational Research, 240, 183-192.

Herrera-Viedma, E., Cabrerizo, F. J., Kacprzyk, J., \& Pedrycz, W. (2014). A review of soft consensus models in a fuzzy environment. Information Fusion, 17, 4-13.

Herrera-Viedma, E., Herrera, F., \& Chiclana, F. (2002). A consensus model for multiperson decision making with different preference structures. IEEE Transactions on Systems Man and Cybernetics - Part A:Systems and Humans, 32(3), 394-402.

Kacprzyk, J., \& Zadrożny, S. (2010). Soft computing and Web intelligence for supporting consensus reaching. Soft Computing, 14, 833-846.

Kacprzyk, J., \& Zadrożny, S. (2016). On a fairness type approach to consensus reaching support under fuzziness via linguistic summaries. IEEE International Conference on Fuzzy Systems (FUZZ-IEEE), 1999-2006.

Kacprzyk, J., Fedrizzi, M., \& Nurmi, H. (1992), Group decision making and consensus under fuzzy preference and fuzzy majority. Fuzzy Sets and Systems, 49(1), 21-31. 
Kacprzyk, J., Fedrizzi, M., \& Nurmi, H. (1997). Soft degrees of consensus under additive preferences and fuzzy majorities. Consensus Under Fuzziness. Springer US.

Kacprzyk, J., Zadrozny, S., \& Ras, Z.W. (2010). How to support consensus reaching using action rules: a novel approach. International Journal of Uncertainty. Fuzziness and Knowledge-Based Systems, 18, 451-470.

Ogryczak, W., Śliwiński, T. (2003). On solving linear programs with the ordered weighted averaging objective. European Journal of Operational Research, 148(1), 80-91.

Palomares, I., Estrella, F. J., Martínez, L., \& Herrera, F. (2014a). Consensus under a fuzzy context: Taxonomy, analysis framework AFRYCA and experimental case of study. Information Fusion, 20, 252-271.

Palomares, I., Martínez, L., \& Herrera, F. (2014b). A consensus model to detect and manage noncooperative behaviors in large-scale group decision making, IEEE Transactions on Fuzzy Systems, 22, 516-530.

Pelta, D. A., \& Yager, R. R. (2010). Decision strategies in mediated multiagent negotiations: An optimization approach, IEEE Transactions on Systems. Man and Cybernetics Part A: Systems and Humans, 40, 635-640.

Quesada, F. J., Palomares, I., Martínez, L. (2014). Managing experts behaviors in large-scale consensus reaching process with uninorm aggregation operators. Applied Soft Computing, 35, 873-887.

Tan, X., Gong, Z. W., Chiclana, F., \& Zhang, N. (2018). Consensus modeling with cost chance constraint under uncertainty opinions. Applied Soft Computing, 67, 721-727.

Yager, R. R. (2001). Penalizing strategic preference manipulation in multi-agent decision making. IEEE Transactions on Fuzzy Systems, 9, 393-403.

Yager, R. R. (2002), Defending against strategic manipulation in uninorm-based multi-agent decision making. European Journal of Operational Research, 141, 217-232.

Zhang, B. W., Dong, Y. C., \& Herrera-Viedma, E. (2019a). Group decision making with heterogeneous preference structures: An automatic mechanism to support consensus reaching. Group Decision and Negotiation, 28, 585-617.

Zhang, G. Q., Dong, Y. C., Xu, Y. F., \& Li, H. Y. (2011). Minimum-cost consensus models under aggregation operators. IEEE Transactions on Systems, Man. Part A: Systems and Humans, 41(6), 1253- 1261.

Zhang, H. H., Gang, K., \& Yi, P. (2019b). Soft consensus cost models for group decision making and economic interpretations. European Journal of Operational Research, 277(3), 964-980.

Zhang, H. J., Dong, Y. C., Chiclana, F., \& Yu, S. (2019c). Consensus efficiency in group decision making: A comprehensive comparative study and its optimal design. European Journal of Operational Research, 275, 580-598.

Zhang, N., Gong, Z., \& Chiclana, F. (2017). Minimum cost consensus models based on random opinions. Expert Systems with Applications, 89, 149-159. 